
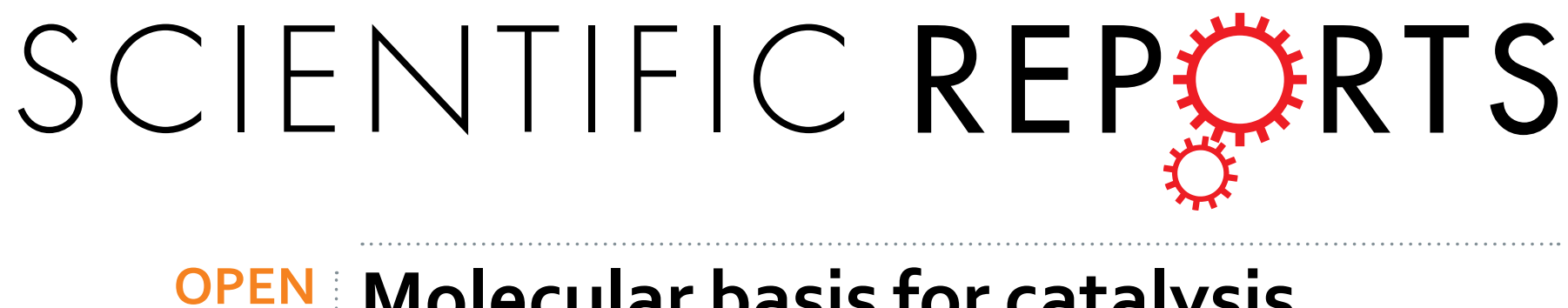

\title{
Molecular basis for catalysis and substrate-mediated cellular stabilization of human tryptophan \\ 2,3-dioxygenase
}

Received: 11 May 2016

Accepted: 26 September 2016

Published: 20 October 2016
Ariel Lewis-Ballester ${ }^{1,{ }^{*}}$, Farhad Forouhar ${ }^{2,{ }^{*}}$, Sung-Mi Kim ${ }^{3}$, Scott Lew ${ }^{2}$, YongQiang Wang ${ }^{3}$, Shay Karkashon ${ }^{1}$, Jayaraman Seetharaman ${ }^{2}$, Dipanwita Batabyal ${ }^{1}$, Bing-Yu Chiang ${ }^{1}$, Munif Hussain ${ }^{2}$, Maria Almira Correia ${ }^{3}$, Syun-Ru Yeh ${ }^{1} \&$ Liang Tong $^{2}$

Tryptophan 2,3-dioxygenase (TDO) and indoleamine 2,3-dioxygenase (IDO) play a central role in tryptophan metabolism and are involved in many cellular and disease processes. Here we report the crystal structure of human TDO (hTDO) in a ternary complex with the substrates $L$-Trp and $\mathrm{O}_{2}$ and in a binary complex with the product $\mathrm{N}$-formylkynurenine (NFK), defining for the first time the binding modes of both substrates and the product of this enzyme. The structure indicates that the dioxygenation reaction is initiated by a direct attack of $\mathrm{O}_{2}$ on the $\mathrm{C}_{2}$ atom of the $L$-Trp indole ring. The structure also reveals an exo binding site for $L$-Trp, located $\sim 2 \AA$ from the active site and formed by residues conserved among tryptophan-auxotrophic TDOs. Biochemical and cellular studies indicate that Trp binding at this exo site does not affect enzyme catalysis but instead it retards the degradation of hTDO through the ubiquitin-dependent proteasomal pathway. This exo site may therefore provide a novel $L$-Trp-mediated regulation mechanism for cellular degradation of $h T D O$, which may have important implications in human diseases.

TDO and IDO are heme proteins that catalyze the oxidative cleavage of $L$-Trp (Supplementary Fig. 1), the first and rate-limiting step of the kynurenine pathway for $L$-Trp catabolism ${ }^{1-7}$. Trp is the least abundant essential amino acid. The majority of dietary $\operatorname{Trp}(\sim 95 \%)$ is metabolized in the liver through this pathway to produce $\mathrm{NAD}^{+}$, while a small amount $(\sim 1 \%)$ is utilized to synthesize serotonin and melatonin through the serotonin pathway. TDO hence plays an important role in controlling the relative Trp flux along the two pathways. Its up-regulation can lead to over-production of neuroactive metabolites as well as serotonin deficiency. In Drosophila, TDO is known as vermilion, responsible for the bright-red eye color, due to its involvement in ommochrome biosynthesis ${ }^{8}$. TDO and IDO have been linked to a variety of human diseases including Alzheimer's, Huntington's, depression-associated anxiety, schizophrenia, and autism ${ }^{9-16}$. Recently, it was discovered that, in addition to the liver, TDO is highly expressed in certain cancer cells, where it plays a critical role in suppressing anti-tumor immunity via activating the aryl hydrocarbon receptor ${ }^{17,18}$. These discoveries have triggered a great deal of new interest in targeting TDO and IDO for drug discovery ${ }^{19-24}$.

TDOs are homo-tetrameric enzymes with $35-45 \mathrm{kD}$ monomers and are well conserved from bacteria to humans (Supplementary Fig. 1). In comparison, IDOs are monomeric enzymes and the sequence conservation between TDO and IDO is much weaker, with 16\% sequence identity between hTDO and human IDO1 (hIDO1) (Supplementary Fig. 1). While the properties of TDO have been studied extensively ${ }^{1,2}$, the detailed dioxygenase mechanism remains poorly understood.

Crystal structures of bacterial TDOs ${ }^{25,26}$, Drosophila TDO (DmTDO) ${ }^{27}$, apo hTDO ${ }^{28}$, and hIDO1 ${ }^{29}$ show that the overall folds and the active sites of TDO and IDO are highly similar, despite their weak sequence conservation.

${ }^{1}$ Department of Physiology and Biophysics Albert Einstein College of Medicine Bronx, NY 10461, USA. ${ }^{2}$ Department of Biological Sciences Northeast Structural Genomics Consortium Columbia University New York, NY 10027, USA. ${ }^{3}$ Departments of Cellular and Molecular Pharmacology, Pharmaceutical Chemistry, and Bioengineering and Therapeutic Sciences, The Liver Center, University of California at San Francisco San Francisco, CA 94158, USA. *These authors contributed equally to this work. Correspondence and requests for materials should be addressed to S.-R.Y. (email: syun-ru.yeh@einstein.yu.edu) or L.T. (email: Itong@columbia.edu) 


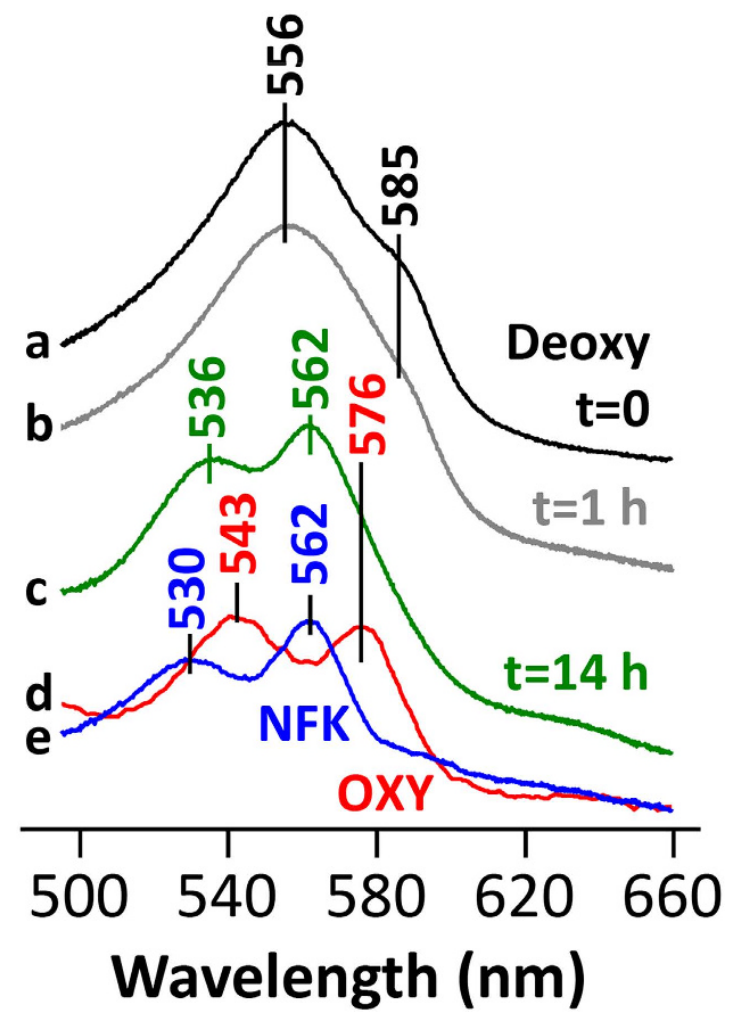

Figure 1. Absorption spectra of hTDO oxygen intermediates. (a) Spectrum of a ferrous hTDO crystal in complex with $L$-Trp, before exposure to $\mathrm{O}_{2}$-saturated solution (black). The spectrum has a band at $556 \mathrm{~nm}$ and a shoulder at $\sim 585 \mathrm{~nm}$, which is characteristic for a five-coordinate high spin heme. (b) Spectrum of the same ferrous hTDO crystal in complex with $L$-Trp, after 1 h exposure to $\mathrm{O}_{2}$ (gray). (c) Spectrum of the same ferrous hTDO crystal in complex with $L$-Trp, after overnight exposure to $\mathrm{O}_{2}(\sim 14 \mathrm{~h})$ (green), with two bands at 536 and $562 \mathrm{~nm}$, indicating the formation of an oxygen intermediate. (d) Typical spectrum of the hTDOTrp- $\mathrm{O}_{2}$ ternary complex obtained by mixing of ferrous hTDO-Trp with air-saturated solution in a stopped-flow system (red). (e) Difference spectrum, (c,d) which likely represents the spectrum of NFK-bound ferrous hTDO (blue). The double peak feature of this spectrum indicates a six-coordinate low spin heme, consistent with the crystallographic observation that NFK is coordinated to the heme iron via its formyl group as the $6^{\text {th }}$ ligand. This spectrum cannot be obtained by mixing hTDO and NFK due to the transient nature of this complex.

The binding mode of $L$-Trp to Xanthomonas campestris TDO (XcTDO, with $34 \%$ sequence identity to hTDO, Supplementary Fig. 1) is known ${ }^{25}$. However, it is unclear how $\mathrm{O}_{2}$ is positioned with respect to $L$ - $\operatorname{Trp}$ in the active site, which is essential for comprehending the dioxygenase mechanism. In addition, the binding mode of the reaction product NFK is not known either.

\section{Results and Discussion}

Structure of hTDO in complex with $L-T r p$ and $\mathrm{O}_{2}$. To define the binding mode of $\mathrm{O}_{2}$ with respect to $L$-Trp and to obtain direct molecular insight into the catalytic mechanism, we prepared crystals of ferrous hTDO in complex with $L$-Trp under anaerobic conditions and then exposed them to an $\mathrm{O}_{2}$-saturated solution at room temperature. The reaction between $\mathrm{O}_{2}$ and Trp in the active site was monitored by microscopic spectroscopy. We observed a large change in the absorption spectrum of the crystal after overnight exposure to $\mathrm{O}_{2}$ (Fig. 1), indicating $\mathrm{O}_{2}$ binding and dioxygenation reaction. The crystal was then flash-frozen for X-ray diffraction data collection. The final atomic model, at $2.5 \AA$ resolution, has excellent agreement with the crystallographic data and the expected bond lengths, bond angles, and other geometric parameters (Table 1). The majority of the residues $(92 \%)$ are in the favored region of the Ramachandran plot. Several other data sets were collected on crystals exposed to $\mathrm{O}_{2}$ using similar protocols, and comparable electron density was observed in the active sites of these crystals as well. In contrast, no electron density for $\mathrm{O}_{2}$ was observed in crystals mounted directly in the glove box, and the structure of hTDO in this binary complex with $L$-Trp is mostly the same as that obtained following $\mathrm{O}_{2}$ exposure (Table 1).

The hTDO monomer has an all $\alpha$-helical structure, and the helices are named $\alpha \mathrm{A}$ through $\alpha \mathrm{L}$, as in XcTDO (Fig. 2a, Supplementary Fig. 1). Three long helices $(\alpha B, \alpha C$, and $\alpha J)$, with 6-10 turns each, are at the center of the hTDO tetramer interface (Fig. 2b-d). Two additional helices, $\alpha \mathrm{E}$ and $\alpha \mathrm{H}$, combine to form another long helix, producing a four-helical bundle in each subunit (Fig. 2a, Supplementary Fig. 2). The heme is located at one end of this four-helical bundle, with the proximal His328 ligand coming from the C-terminal region of helix $\alpha$ J (Fig. 2a). A helix-loop-helix segment $\left(\alpha \mathrm{H}_{1}-\alpha \mathrm{H}_{2}\right)$ is located near the other end of this bundle (Fig. 2a), which is also present in DmTDO but not XcTDO (Supplementary Fig. 3). 


\begin{tabular}{|c|c|c|}
\hline & $\begin{array}{l}\text { Crystal exposed to } \mathrm{O}_{2} \text { (Ternary } \\
\text { complex with } L \text {-Trp and } \mathrm{O}_{2} \text { and } \\
\text { binary complex with NFK) }\end{array}$ & $\begin{array}{l}L \text {-Trp ferrous binary } \\
\text { complex (anaerobic) }\end{array}$ \\
\hline \multicolumn{3}{|l|}{ Data collection } \\
\hline Space group & $P 2_{1} 2_{1} 2$ & $P 2_{1} 2_{1} 2$ \\
\hline \multicolumn{3}{|l|}{ Cell dimensions } \\
\hline$a, b, c(\AA)$ & $143.6,154.0,87.8$ & $144.4,153.6,88.2$ \\
\hline$\alpha, \beta, \gamma\left({ }^{\circ}\right)$ & $90,90,90$ & $90,90,90$ \\
\hline Resolution $(\AA)$ & $48.3-2.5(2.54-2.5)^{*}$ & $35.1-2.44(2.54-2.44)^{\star}$ \\
\hline$R_{\text {merge }}$ & $0.062(0.552)$ & $0.107(0.792)$ \\
\hline$I / \sigma I$ & $37.3(4.3)$ & $32.0(3.3)$ \\
\hline Completeness (\%) & $99.9(98.5)$ & $100(100)$ \\
\hline Redundancy & $6.8(5.8)$ & $8.5(7.9)$ \\
\hline \multicolumn{3}{|l|}{ Refinement } \\
\hline Resolution $(\AA)$ & $34-2.5(2.53-2.5)$ & $35.1-2.44(2.47-2.44)$ \\
\hline No. reflections & 68,151 & 72,907 \\
\hline$R_{\text {work } /} R_{\text {free }}$ & $15.9 / 23.0$ & $16.8 / 23.0$ \\
\hline No. atoms & 12,070 & 12,061 \\
\hline Protein & 11,342 & 11,319 \\
\hline Ligand/ion & 288 & 268 \\
\hline Water & 436 & 474 \\
\hline \multicolumn{3}{|l|}{ B-factors } \\
\hline Protein & 45.4 & 44.8 \\
\hline Ligand/ion & 48.6 & 43.6 \\
\hline Water & 45.3 & 48.3 \\
\hline \multicolumn{3}{|l|}{ R.m.s deviations } \\
\hline Bond lengths $(\AA)$ & 0.011 & 0.008 \\
\hline Bond angles $\left({ }^{\circ}\right)$ & 1.3 & 1.1 \\
\hline
\end{tabular}

Table 1. Data collection and refinement statistics. One crystal was used for each data collection. ${ }^{\star}$ Highest resolution shell is shown in parenthesis.

Binding modes of the substrates $L-\operatorname{Trp}$ and $\mathrm{O}_{2}$. The crystallographic analysis revealed that the active sites of the four subunits of the hTDO tetramer are in different states in terms of the catalytic conversion of $L$-Trp, giving us several snapshots along the reaction coordinate. Clear electron density was observed for $L-\operatorname{Trp}$ and $\mathrm{O}_{2}$ in subunits A (Fig. 3a) and B, allowing the definition of the relative regioorientation of the two substrates prior to the reaction for the first time. The $\mathrm{O}_{2}$ substrate is coordinated to the heme iron as the sixth ligand. The Fe-O-O moiety is slightly bent (Fig. 3b), with a Fe-O-O angle of $\sim 150^{\circ}$. The angle is significantly larger than that expected for a typical ferrous iron bound neutral dioxygen $\left(\angle \mathrm{Fe}-\mathrm{O}-\mathrm{O}=120^{\circ}\right)^{30}$, consistent with the $\left[\mathrm{Fe}^{3+}-\mathrm{O}_{2}^{-}\right]$electronic configuration revealed by earlier resonance Raman studies ${ }^{31}$. The terminal oxygen atom is situated next to the plane of the $L$-Trp indole ring, close to its $\mathrm{C}_{2}(2.9 \AA$ distance $)$ and $\mathrm{N}_{1}(2.5 \AA)$ atoms. Intriguingly, the electron density for the terminal oxygen atom is connected to these two atoms of the indole ring in subunit $A$, although the origin of this connection is not clear and this connection is much weaker in subunit B. We tried different conformational states for $L$-Trp but none of them could satisfactorily explain the density connecting $\mathrm{O}_{2}$ to the indole. The terminal oxygen also has strong hydrogen-bonding interactions with the main-chain amide of Gly152 (2.5 $\AA$ ) in the $\mathrm{DE}$ loop (connecting helices $\alpha \mathrm{D}$ and $\alpha \mathrm{E}$ ). This oxygen is $4.4 \AA$ from the main-chain ammonium ion of the $L$ - $\operatorname{Trp}$ substrate, although earlier experimental and QM/MM studies suggested that they are within hydrogen-bonding distance ${ }^{32-34}$. These interactions strategically position $\mathrm{O}_{2}$ next to $L$-Trp for the catalysis.

The recognition of the $L$-Trp substrate by hTDO (Fig. 3 b) is similar to that by $\mathrm{XcTDO}^{25}$ (Fig. $3 \mathrm{c}$ ), as most of the residues in the active site region of the two enzymes are conserved (Supplementary Fig. 1). His76 is hydrogen-bonded to the $\mathrm{N}_{1}$ atom of $L$-Trp, although this interaction is not essential for TDO catalysis ${ }^{35,36}$ and this residue is replaced by Ser in IDO (Supplementary Fig. 1). The JK loop, with a $\beta$-reverse turn structure at its tip formed by a highly conserved GTGG motif (Supplementary Fig. 1), covers the active site (Fig. 3b). The hydroxyl group of the Thr side chain in this motif is hydrogen-bonded to the ammonium ion of $L$-Trp and the main-chain carbonyl oxygen of Ala150, which may also help organize the active site for catalysis.

Binding mode of the product NFK. The electron density in the active site of subunit C (Fig. 4a) and subunit D (Fig. 4b) is consistent with the product NFK, allowing us to define for the first time the binding mode of this product to TDO. The pyrrole ring of the Trp side chain has opened, but good electron density was observed only for the remaining six-membered ring and its $\mathrm{N}$-formyl group, which is coordinated to the heme iron. In contrast, the indole ring of $L$-Trp does not fit well into the density, and the ring cannot explain the strong density connection to the iron (Fig. 4a,b). The JK loop is ordered in subunit C but disordered in subunit D (Fig. 4c). The rest of the structures of the two subunits are similar to each other (Supplementary Fig. 2). The observation that two subunits of the tetramer are trapped in the $\mathrm{O}_{2}$ and Trp bound state while the other two are in the product-bound 

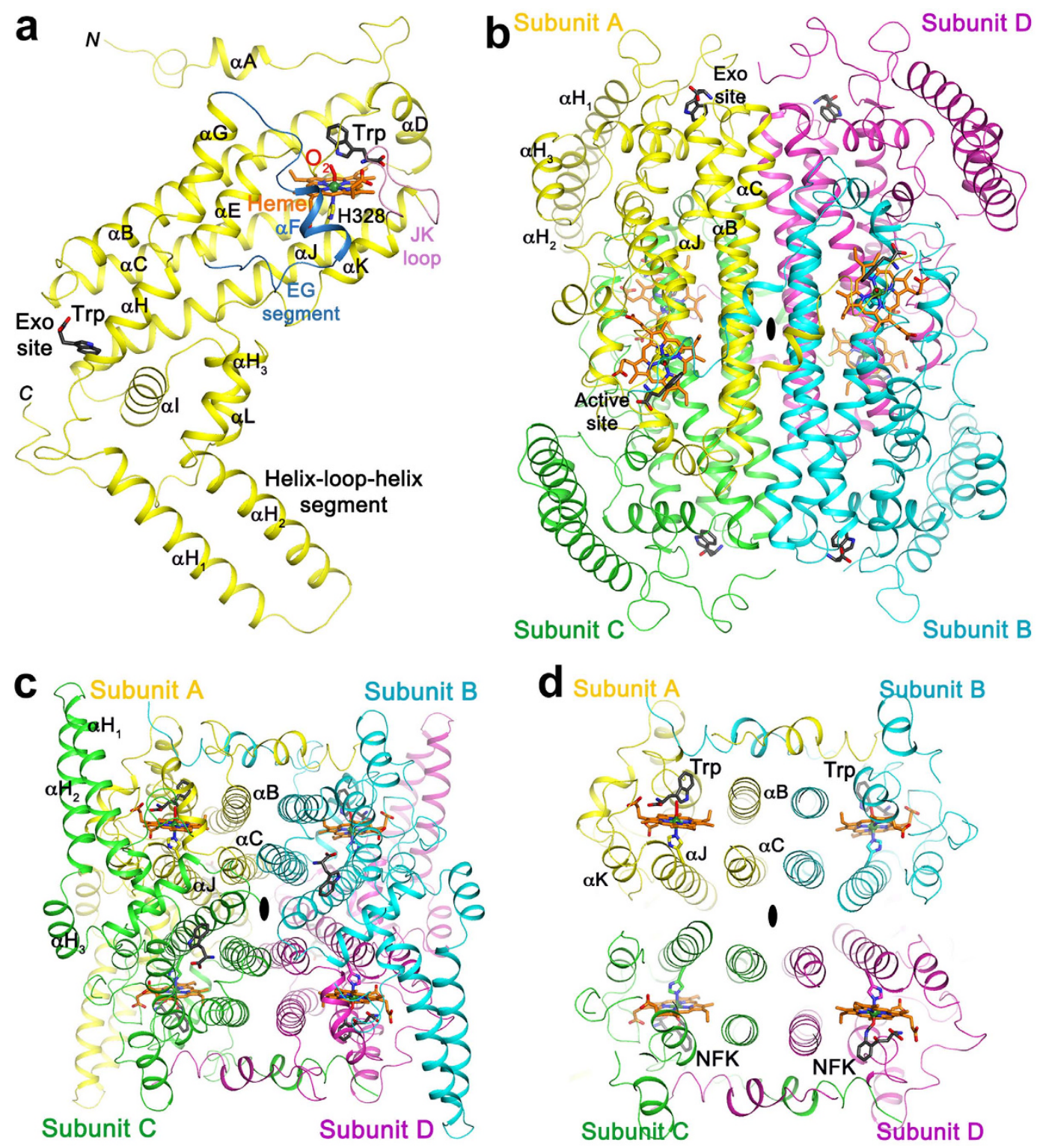

Figure 2. The structure of human TDO (hTDO). (a) Schematic representation of the structure of the hTDO monomer in a ternary complex with $L$-Trp and $\mathrm{O}_{2}$. Heme (in orange), $L$-Trp in the active site and the exo site (black), and oxygen (red) are shown as stick models. (b) Schematic representation of the hTDO tetramer, viewed down the two-fold axis (black oval) relating subunits A and B. The four subunits (A, B, C, and D) are colored in yellow, cyan, green and magenta, respectively. (c) Schematic representation of the hTDO tetramer viewed down the two-fold axis (black oval) relating subunits A and D. (d) A thin section of the structure of the hTDO tetramer, centered around the four hemes in the active sites, showing the three helices in each subunit that are in the tetramer interface. All structure figures were produced with the program PyMOL (www.pymol.org).

state seems to indicate that the dioxygenase reaction is cooperative, but so far no cooperativity is observed in free solution reactions of hTDO. Nonetheless, EPR and Mössbauer studies of a bacterial TDO do support that the four heme sites in TDO are not equivalent ${ }^{37}$.

Implications for hTDO catalytic mechanism. The crystallographic analyses have allowed us to directly visualize both the substrate and product complexes of hTDO. The structural data, together with earlier spectroscopic and computational studies ${ }^{31-33}$, support a two-step ferryl-based dioxygenation mechanism (Fig. 5a). The first step of the reaction is initiated by radical addition of the heme-iron-bound dioxygen to $\mathrm{C}_{2}$ of $L$-Trp to generate a ferryl and Trp-epoxide intermediate, via a 2-indolenylperoxo transition state. An alternative, electrophilic addition of $\mathrm{O}_{2}$ to $L$-Trp in the first step of the reaction has also been proposed ${ }^{38-40}$, but QM/MM calculations favor radical addition mechanism ${ }^{31-33}$. In the second step, protonation of the epoxide by the ammonium ion of $L$-Trp opens the epoxide ring and triggers the addition of the ferryl-oxygen to $\mathrm{C}_{2}$, ultimately leading to the breakage of the $\mathrm{C}_{2}-\mathrm{C}_{3}$ bond and the formation of the NFK product. The formyl group of NFK is coordinated to the iron atom 
a

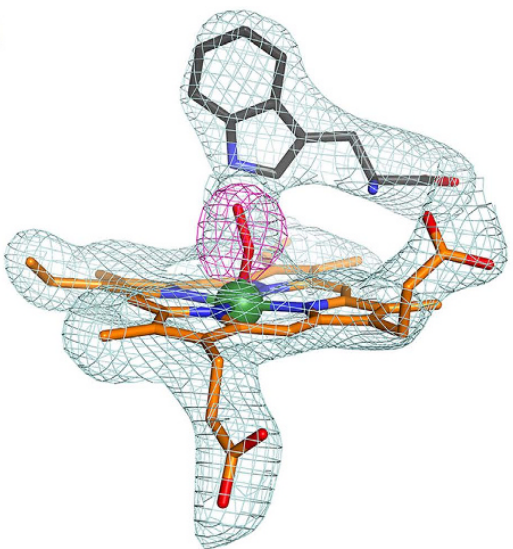

b

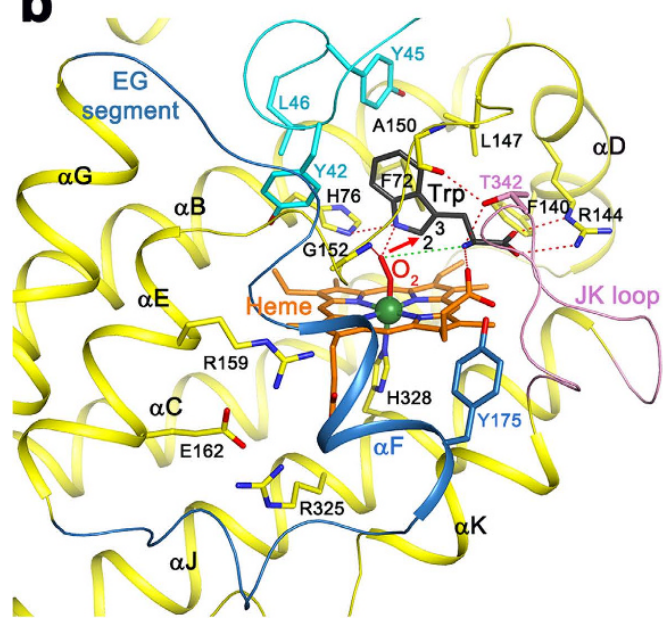

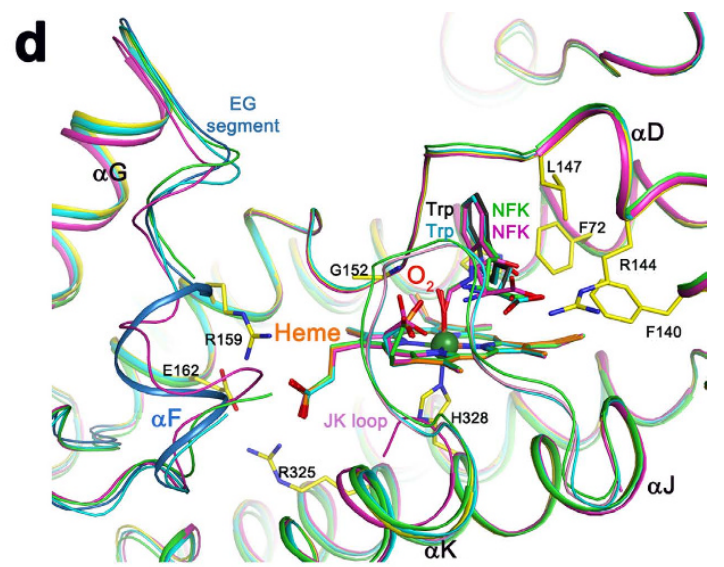

C

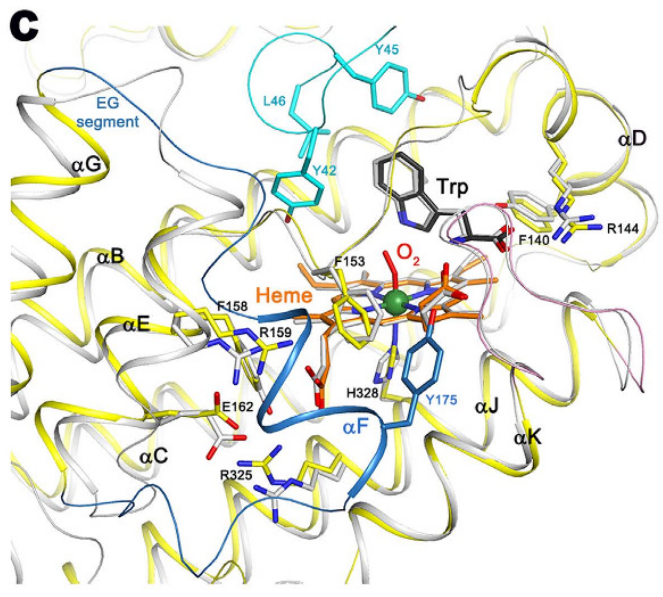

Figure 3. Molecular basis for substrate recognition by hTDO. (a) Omit $\mathrm{F}_{\mathrm{o}}-\mathrm{F}_{\mathrm{c}}$ map (light blue) at $2.5 \AA$ resolution for heme, $L$-Trp and $\mathrm{O}_{2}$ in the active site of subunit $\mathrm{A}$, contoured at $3 \sigma$. Omit $\mathrm{F}_{\mathrm{o}}-\mathrm{F}_{\mathrm{c}}$ map for $\mathrm{O}_{2}$ (magenta), contoured at $5 \sigma$. (b) Schematic drawing showing the active site of hTDO in the ternary complex with $L$-Trp and $\mathrm{O}_{2}$. The segment in cyan is from subunit $\mathrm{B}$ of the tetramer, which forms a part of the binding pocket for the Trp side chain. The ionic interaction between the terminal oxygen atom and the ammonium ion of $L$ - $\operatorname{Trp}$ is indicated by the dashed line in green. The attack of $\mathrm{O}_{2}$ on the $\mathrm{C}_{2}$ atom of $L$-Trp is indicated with the red arrow. (c) Overlay of the active site structures of hTDO (in color) and XcTDO (in gray). Residues Tyr42, Tyr45 and Leu46 from the N-terminal segment of the neighboring subunit are shown in cyan. (d) Overlay of the active site structures of the four subunits of hTDO. Conformational differences for the EG segment and the disordering the JK loop in two subunits are visible.

after the reaction. The disordering of the JK loop allows NFK to diffuse out of the active site, thereby enabling the binding of a new $L$-Trp for the next round of the reaction.

Besides the disordering of the JK loop in subunit $\mathrm{D}$, the structures of the four subunits in the immediate active site region are highly similar to each other (Fig. 3d). On the other hand, large conformational differences in the segment connecting $\alpha E$ and $\alpha G$ (EG segment) are observed in the four subunits (Fig. 3d). This segment contains a short helix $(\alpha \mathrm{F})$ in subunit A (Fig. 3b), but is partly disordered in subunits B and C (Fig. 3d), and is a loop in subunit D (Fig. 4c), where its conformation is partly stabilized by crystal packing. The Tyr 175 side chain in this segment is positioned near the JK loop in subunit A (Fig. 3b), but in subunit D it would clash with the JK loop if the loop were ordered (Fig. 4c). In comparison, the EG segment in XcTDO is positioned further away from the active site due to a deletion in this region (Fig. 3c and Supplementary Fig. 1). On the other hand, the EG segment, as well as the JK loop and helix $\alpha \mathrm{K}$ are disordered in the apo hTDO structure ${ }^{28}$ (Supplementary Fig. 3), due to the absence of the heme and substrates.

To assess the functional importance of the EG segment, we created the Y175G mutant and compared its activity to that of the wild-type (WT) enzyme with stopped-flow measurements (Supplementary Figs 4 and 5). As summarized in Fig. 5, the mutation led to a 6-fold slower multiple turnover velocity (Fig. 5b). In addition, pre-incubation of the Y175G mutant with $8 \mathrm{mM}$ NFK retards the formation of the ternary complex by $\sim 100$-fold (Fig. 5c) and impedes Trp binding (Fig. 5d). Overall, the data suggest that the EG segment in hTDO plays a critical role in promoting NFK release, thereby allowing Trp binding during multiple turnover. 

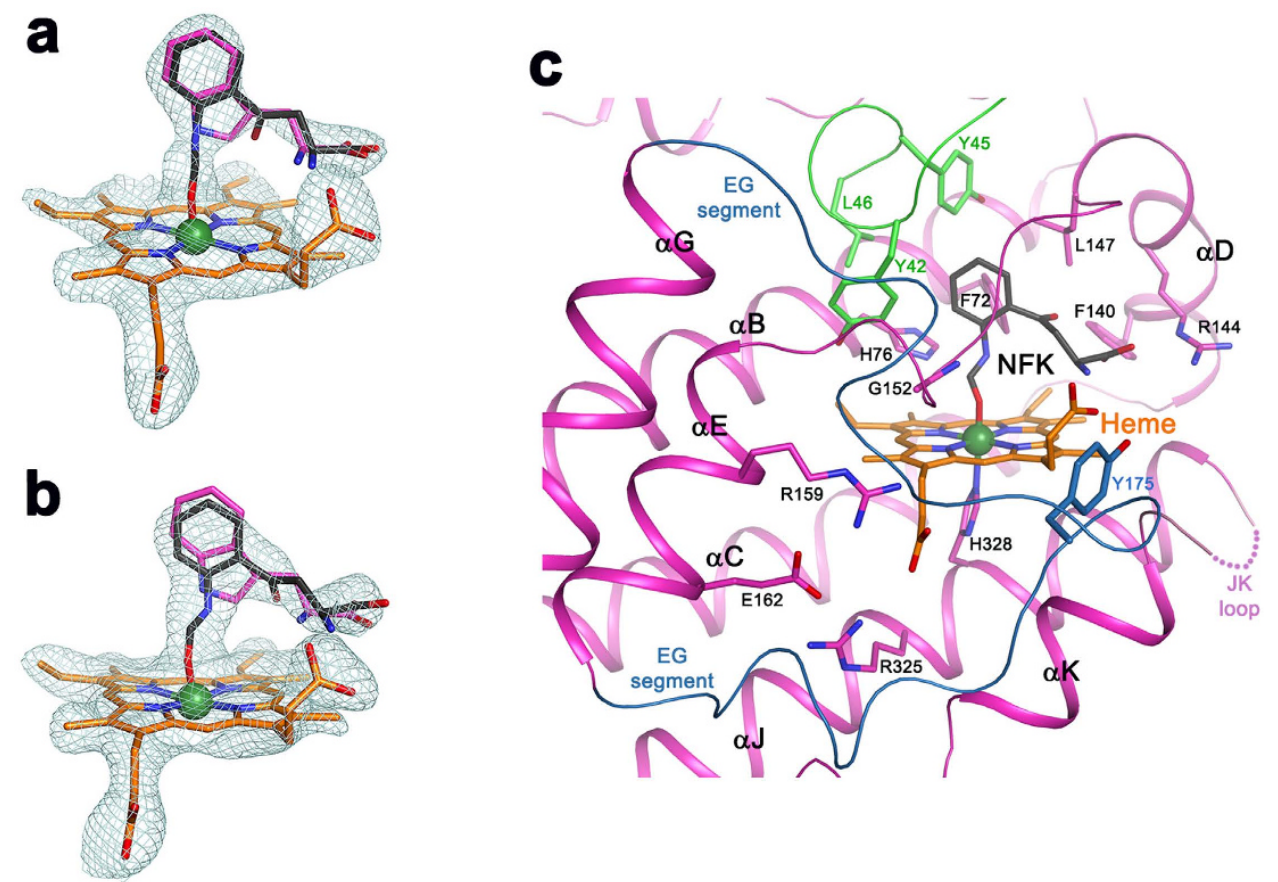

Figure 4. Molecular basis for NFK recognition by hTDO. (a) Omit $F_{o}-F_{c}$ map at $2.5 \AA ̊$ resolution for heme and NFK in the active site of subunit $\mathrm{C}$, contoured at $2.5 \sigma$. The binding mode of Trp in subunit $\mathrm{A}$ is shown as a reference. (b) Omit $\mathrm{F}_{\mathrm{o}}-\mathrm{F}_{\mathrm{c}}$ map at $2.5 \AA$ resolution for heme and NFK in the active site of subunit $\mathrm{D}$, contoured at $2.5 \sigma$. (c) Schematic drawing showing the active site of hTDO in the binary complex with NFK in subunit D, where the JK loop is disordered (dotted curve).

An exo site for $L$-Trp binding in hTDO. The structure revealed that a second $L$-Trp molecule is bound to each hTDO subunit, with well-defined electron density (Fig. 6a). This exo site is located at the other end of the four-helical bundle and is $\sim 42 \AA$ from the active site (Fig. 2a). The side chain of this $L$-Trp is sandwiched between Trp208 (helix $\alpha \mathrm{H}$ ) and Pro213, and is in direct contact with several other hydrophobic residues (Fig. 6b). The main-chain carboxylate group has bidentate ion-pair interactions with the side chain of Arg211 $(\alpha \mathrm{H})$. The ammonium ion of $L$-Trp is positioned near the main-chain carbonyl of Arg103 and the side chain of Glu105, which also has ion-pair interactions with Arg303.

Residues forming this novel exo site are well conserved among tryptophan-auxotrophic TDOs, but they are generally poorly conserved in other orthologs (Supplementary Fig. 1). This binding site does not exist in XcTDO, due to side-chain substitutions as well as main-chain conformational differences. Nonetheless, a second binding site for $L$-Trp was observed in XcTDO at the tetramer interface ${ }^{25}$ (Supplementary Fig. 3), while the exo site in hTDO is located within each subunit.

We next carried out isothermal titration calorimetry (ITC) experiments to verify the presence of two $L$-Trp binding sites in hTDO. Two transitions were observed, corresponding to association constants of $2.06 \pm 1.34 \times 10^{6} \mathrm{M}^{-1}\left(K_{\mathrm{d}} \sim 0.5 \mu \mathrm{M}\right)$ and $1.84 \pm 0.04 \times 10^{4} \mathrm{M}^{-1}\left(K_{\mathrm{d}} \sim 54 \mu \mathrm{M}\right)$ (Fig. $6 \mathrm{c}$ ), which we assigned to the exo site and the active site, respectively. To confirm this assignment, we generated a double mutant W208V/R211L (referred to as the WR mutant hereafter) and showed that the mutation abolished the high affinity site without significantly perturbing the low affinity site (Fig. 6d). Overall, the ITC data revealed that the exo site exhibits 100 -fold higher affinity for $L$-Trp than the active site, and that the WR double mutation is sufficient to disrupt $L$-Trp binding to the exo site.

We also examined whether the exo site has any effect on the catalytic activity of hTDO. While it might be expected that the exo site can regulate hTDO catalysis indirectly by modulating the organization of the four helical bundle (Fig. 2a), our kinetic data indicate that a triple mutant, E105L/W208V/R211L (EWR mutant), had only a minor defect in catalysis as compared to the wild-type enzyme (Fig. 6e).

The exo site regulates hTDO cellular stability. Mouse TDO (mTDO) is one of the most rapidly degraded hepatic proteins, with a half-life $\left(\mathrm{t}_{1 / 2}\right)$ of $\sim 2.5 \mathrm{~h}$, versus a mean $\mathrm{t}_{1 / 2}$ of $\sim 2-3$ days for total liver protein ${ }^{41}$. This unusually short lifespan is consistent with the large number (15) of mTDO ubiquitination sites ${ }^{42}$, which probably enables its rapid removal via ubiquitin (Ub)-dependent proteasomal degradation (UPD). A second Trp-binding site in mTDO was previously proposed based on in vivo and in vitro studies ${ }^{41}$. In addition, it was shown that binding of $L$-Trp, or its $\alpha$-methyl analog ( $\alpha \mathrm{MTrp}$ ), to this site not only stabilizes the enzyme against heat, urea or proteases, but also reduces its hepatic degradation.

We hypothesized that the exo site observed in the current hTDO structure is equivalent to the second site functionally identified in $\mathrm{mTDO}$ and that binding of $L$-Trp to the exo site stabilizes hTDO against UPD, thereby regulating its biological lifespan. To test this hypothesis, we first carried out ${ }^{35} \mathrm{~S}$-pulse-chase analyses 
a
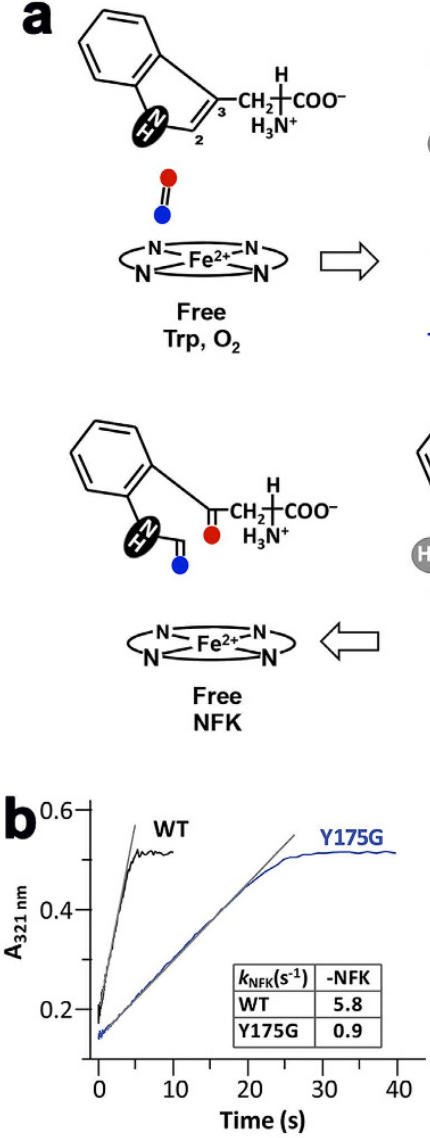
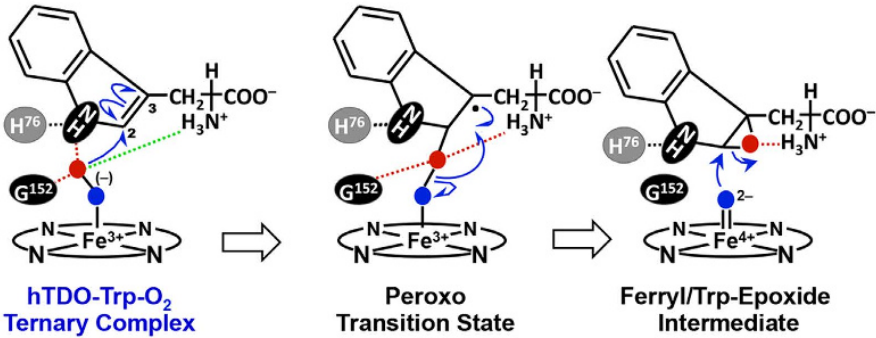

Transition State Intermediate
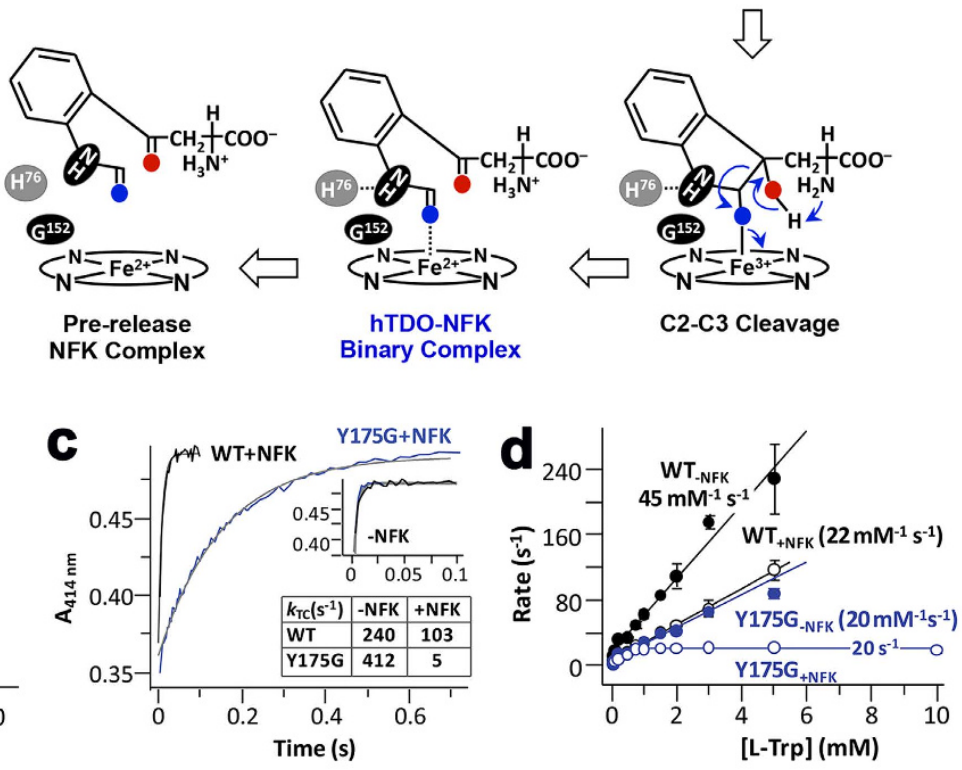

Figure 5. Catalytic mechanism of hTDO. (a) A two-step, ferryl-based mechanism for the dioxygenation of $L$ Trp. The two complexes observed in the current crystal are labeled in blue. The electrostatic interaction between the terminal oxygen of $\mathrm{O}_{2}$ and the main-chain ammonium ion of $L$-Trp is indicated with the dashed lines in green. Modified from earlier publications $\mathrm{s}^{31-33}$. (b) Stopped-flow kinetics showing that the multiple turnover activity of the enzyme is reduced by the Y175G mutation. The production of NFK was monitored by absorbance at $321 \mathrm{~nm}$. (c) Stopped-flow kinetics showing that the presence of NFK $(8 \mathrm{mM})$ retarded Trp- $\mathrm{O}_{2}$ binding to the deoxy enzymes to form the ternary complexes, especially for the Y175G mutant. TC: ternary complex, monitored by absorbance at $414 \mathrm{~nm}$. The inset shows the kinetic traces obtained in the absence of NFK. (d) Trp binding kinetics and its retardation by NFK. The Trp binding rate of the Y175G mutant is linear with [Trp], leading to a $k_{\text {on }}$ of $20 \mathrm{mM}^{-1} \mathrm{~s}^{-1}$. In the presence of $8 \mathrm{mM} \mathrm{NFK}$, the same $k_{\text {on }}$ was observed at low [Trp]; as the [Trp] was raised above $1 \mathrm{mM}$, the rate leveled off at $20 \mathrm{~s}^{-1}$, as the reaction was rate-limited by NFK release. The data in (b) and (c) were taken from Supplementary Figs 4 and 5.

of a full-length $\mathrm{His}_{6}$-tagged hTDO protein expressed in a human liver HepG2 cell culture. We found that the inclusion of $\alpha \mathrm{MTrp}$, which primarily binds to the exo site (as its $\alpha$-methyl group has steric clashes with the heme in the active site), in the culture media indeed increased the in vivo $t_{1 / 2}$ of hTDO protein by $\sim$-fold (from 90 to $172 \mathrm{~min}$ ), based on total radioactivity (Fig. 7a) as well as SDS-PAGE/fluorographic analyses of the pulled-down parent $(47 \mathrm{kD})$ and ubiquitinated $(>56 \mathrm{kD})$ hTDO species (Fig. $7 \mathrm{~b})$. In contrast, the EWR mutant was not stabilized by $\alpha M T r p$, confirming that the stabilization is due to $\alpha M \operatorname{Trp}$ binding to the exo site. We used $\alpha M \operatorname{Trp}$ rather than $L$-Trp in these experiments as HepG2 cells require aerobic culture for viability, and exogenous $L$-Trp is readily consumed by the over-expressed hTDO under these conditions. Marked hTDO stabilization was also observed in HepG2 cells upon inclusion of the proteasomal inhibitor MG132 $(10 \mu \mathrm{M})$, thereby attesting to its cellular UPD (data not shown). Together, these data verify that UPD is a major pathway for cellular hTDO disposal and that $\alpha$ $\mathrm{MTrp} / L$-Trp binding to the exo site can enhance its intracellular stability by reducing its UPD.

To identify the hTDO ubiquitination sites, we incubated the enzyme with two E2/E3 Ub-ligase complexes that are major participants in the UPD of some hepatic proteins ${ }^{43,44}$ and identified 15 ubiquitinated Lys residues by Ub-remnant profiling and LC-MS/MS analyses (Table 2). While similar proteomic analyses of mouse liver extracts identified ten of these sites in mTDO previously ${ }^{42}, 5$ of the sites identified here (K17, K110, K185, K194 and K259) are entirely new (Table 2). In addition, we found that the three E2/E3 complexes have different hTDO-ubiquitination efficiencies: Ubc7/gp78 > UbcH5a/CHIP > Ubc7/Hrd1 (Fig. 7c).

Molecular recognition of hepatic cytochromes $\mathrm{P} 450$ by these E2/E3 Ub-ligases involves electrostatic interactions between positively charged E2/E3-domains and negatively charged surface clusters of phosphorylated Ser/Thr and acidic Asp/Glu-residues. Lys residues within such clusters in P450 were shown to be predominantly 

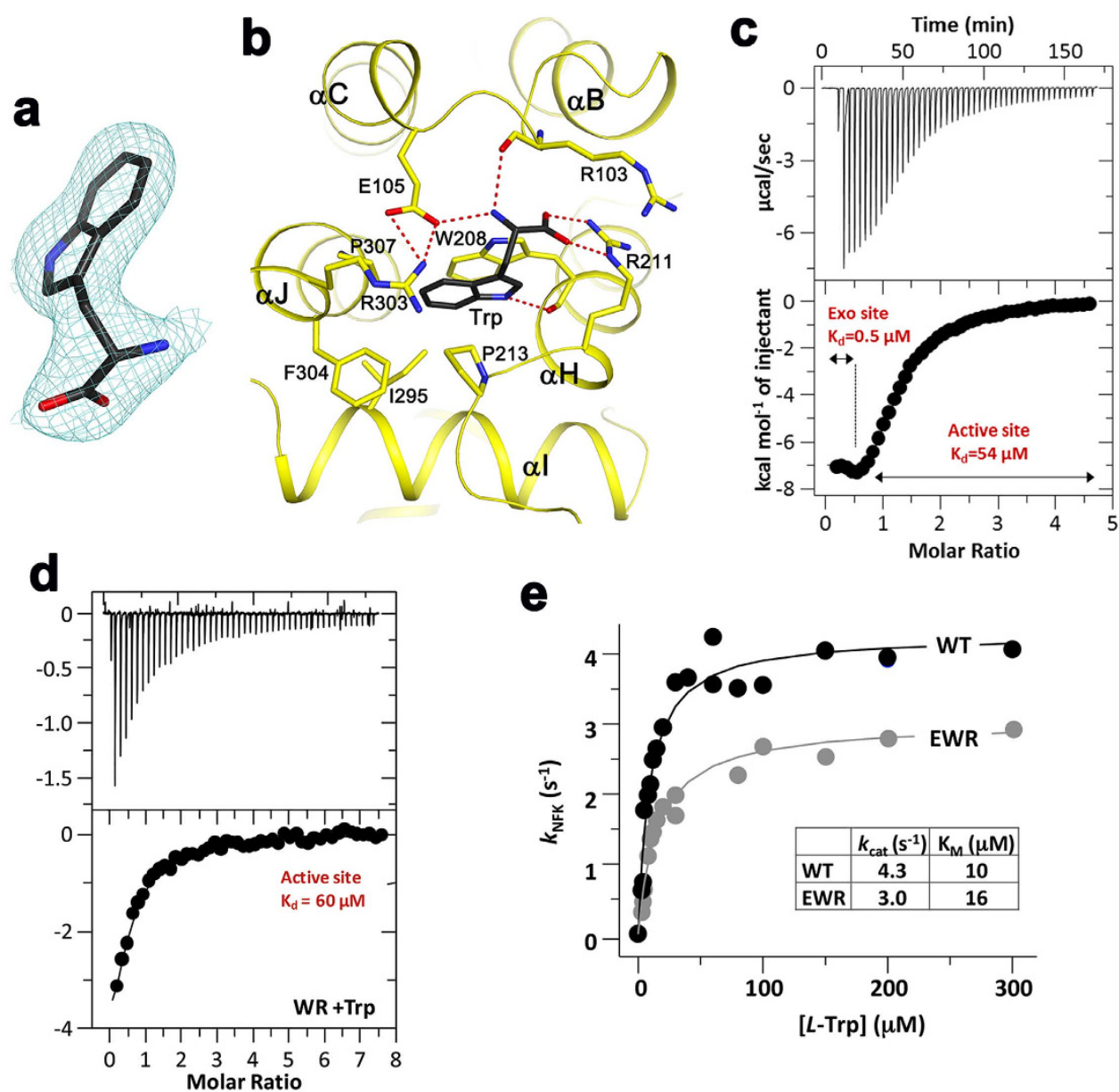

Figure 6. A novel exo site for $L$-Trp in hTDO. (a) Omit $\mathrm{F}_{\mathrm{o}}-\mathrm{F}_{\mathrm{c}}$ map at $2.5 \AA$ resolution for $L$-Trp in the exo site of subunit A, contoured at $4.5 \sigma$. (b) Schematic drawing showing the interactions between $L$-Trp and the exo site. Hydrogen-bonding and ionic interactions are indicated with the dashed lines in red. (c) ITC data for wild-type hTDO demonstrating the presence of two $L$-Trp binding sites, with association constants of $2.06 \pm 1.34 \times 10^{6} \mathrm{M}^{-1}\left(K_{\mathrm{d}} \sim 0.5 \mu \mathrm{M}\right)$ and $1.84 \pm 0.04 \times 10^{4} \mathrm{M}^{-1}\left(K_{\mathrm{d}} \sim 54 \mu \mathrm{M}\right)$. (d) Representative ITC titration of the WR exo site mutant with Trp in the ferric state, showing binding only to the active site. (e) MichaelisMikenten plots of the wild type (WT) and EWR mutant reactions showing that the exo site does not significantly perturb the $k_{\text {cat }}$ and $K_{\mathrm{m}}$ of the enzyme.

targeted for ubiquitination ${ }^{44}$. The 15 hTDO ubiquitination sites are distributed throughout the surface of the monomer (Fig. 7d) and tetramer. Intriguingly, the helix-loop-helix segment of hTDO is rich in negatively-charged Glu residues and 5 of its 15 identified ubiquitination sites are located within this region (Fig. 7d). Conceivably, this segment is important for the molecular recognition of hTDO by these E2/E3 complexes, and the subsequent ubiquitination of Lys residues in this segment is required for UPD. As the exo site is located close to this helix-loop-helix segment (Fig. 2a), this offers a plausible molecular mechanism for this site to regulate hTDO ubiquitination and UPD. Our studies have thus provided novel mechanistic insights into the cellular regulation of hTDO degradation by its $L$-Trp substrate, which may have important implications for the role of this enzyme in human diseases. They also offer invaluable structural basis for future QM/MM studies to further refine the proposed ferryl-based dioxygenation mechanism.

\section{Methods}

Protein expression and purification. Expression of hTDO was performed as described elsewhere ${ }^{4,36}$. To ensure homogeneity of the sample, the protein was oxidized with potassium ferricyanide and immediately loaded on a G25 column pre-equilibrated with $50 \mathrm{mM}$ Tris ( $\mathrm{pH} 7.4$ ) and $150 \mathrm{mM} \mathrm{NaCl}$. The protein collected was flash frozen and stored in $-80^{\circ} \mathrm{C}$ until use.

Protein crystallization. The binary complex of hTDO was crystallized using the under-oil microbatch method under anaerobic conditions inside a glove box at $18^{\circ} \mathrm{C} .3 \mu \mathrm{l}$ of hTDO $(45 \mathrm{mg} / \mathrm{ml})$ in $50 \mathrm{mM}$ Tris (pH 8.0), containing $150 \mathrm{mM} \mathrm{NaCl}$ and $10 \mathrm{mM} \mathrm{L}$-Trp, was reduced with 2 -fold molar excess of sodium dithionite. It was then mixed with $3-6 \mu \mathrm{L}$ of a precipitant solution, containing $50 \mathrm{mM}$ sodium citrate (pH 5.6), 5\% (w/v) PEG 3350, 

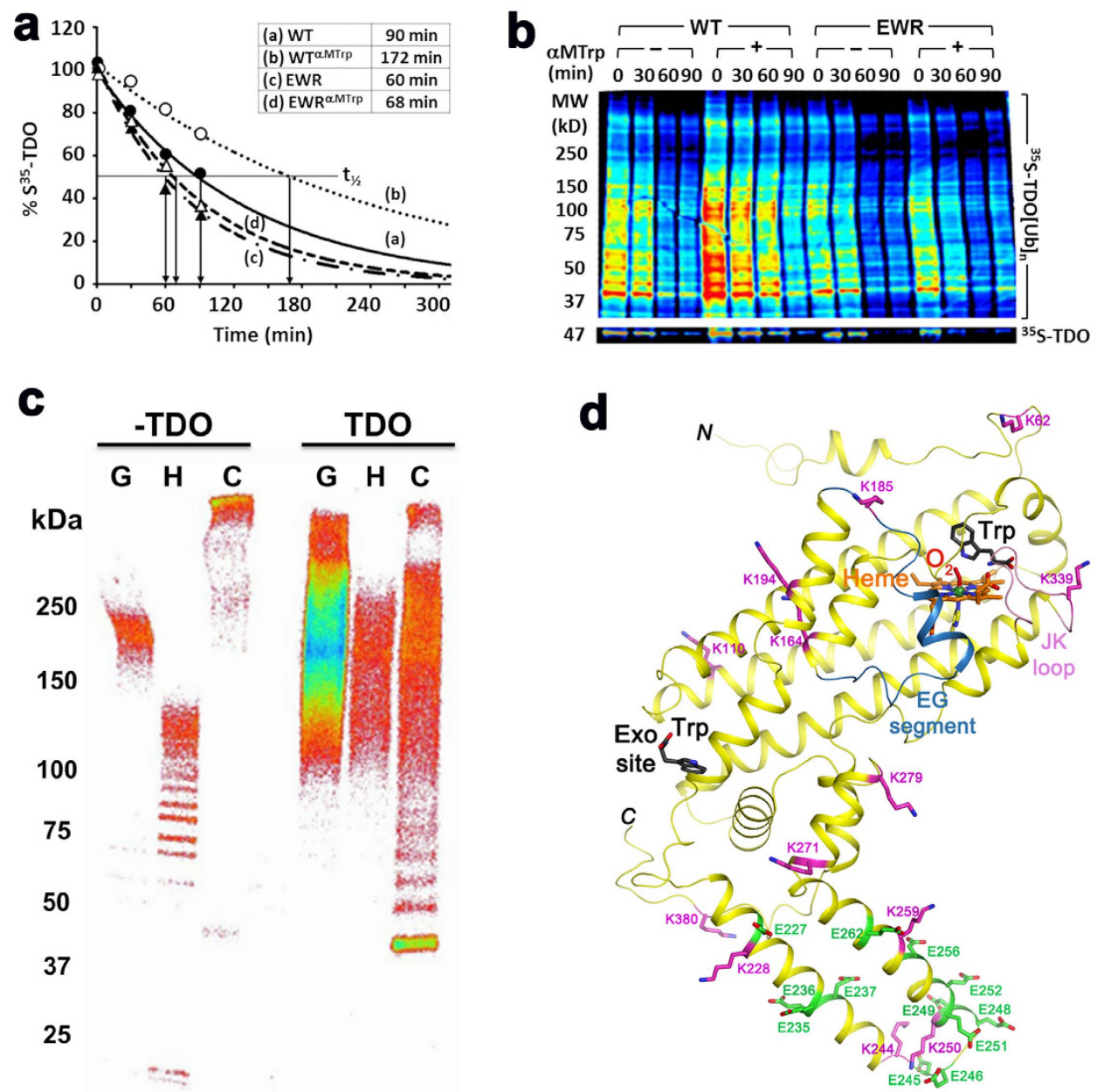

Figure 7. The exo site is required for $L$-Trp-elicited hTDO protein stabilization against UPD.

(a) ${ }^{35}$ S-pulse-chase analyses of hTDO protein degradation in HepG2 cells, showing $\alpha$ MTrp-stabilization of the WT hTDO protein but not its EWR mutant with the disrupted exo site. (b) SDS-PAGE/fluorographic analyses of the ${ }^{35} \mathrm{~S}$-hTDO species pulled-down from the pulse-chase experiments. A shorter time-exposure of the parent ${ }^{35} \mathrm{~S}$-TDO species $(47 \mathrm{kD})$ in the same gel is shown in the bottom panel. Color code wheel: Red $>$ orange $>$ yellow $>$ green $>$ blue $>$ indigo $>$ violet. $\alpha$ MTrp greatly stabilizes hTDO by reducing the degradation of its parent and ubiquitinated species in cells expressing the WT protein, but not the EWR mutant. (c) In vitro ubiquitination of hTDO by three E2/E3 systems - G: Ubc7/gp78; H: Ubc7/Hrd1; C: UbcH5a/CHIP/ Hsc70/Hsp40. Experimental details of the ubiquitination reactions are described in ref. 43. Color code wheel: Violet $>$ indigo $>$ blue $>$ green $>$ yellow $>$ orange $>$ red $>$. The comparative studies demonstrate that among the three complexes tested, the Ubc7/gp78 complex is the most efficient ubiquitination system for hTDO. (d) Structure of hTDO monomer showing the 13 ubiquitination K-sites (magenta) and the negatively charged glutamate residues (green) in the helix-loop-helix segment. Two additional K-sites identified in this study, K17 and $\mathrm{K} 37$, are not shown. $\mathrm{K} 17$ is not included in the expression construct, and $\mathrm{K} 37$ is located in a disordered region.

and $2 \%(\mathrm{w} / \mathrm{v})$ Tacsimate ( $\mathrm{pH}$ 5). Crystals were harvested after 3-5 days. The optical absorption spectra were taken to ensure the crystals were in a fully reduced deoxy state.

Microscopic spectroscopy. Crystals of the Trp-bound binary complex were soaked in an $\mathrm{O}_{2}$-saturated precipitant solution supplemented with $20 \%(\mathrm{v} / \mathrm{v})$ ethylene glycol at room temperature. The in-crystal reactions were monitored with a customized Raman microscope system (Labram HR from Horiba Jobin Yvon). Very little change in the spectrum was observed within an hour. Overnight $(\sim 14 \mathrm{~h})$ incubation led to a new spectrum indicating the formation of an oxygen intermediate. The intermediate crystals were flash-frozen in liquid nitrogen for structure determination. The crystal was annealed at the beamline before data collection due to ice accumulation.

Data collection and structure determination. X-ray diffraction data were collected at the X4A and X4C beamlines of National Synchrotron Light Source (NSLS). The diffraction images were processed with the program $\mathrm{HKL}^{45}$, and the data processing statistics are summarized in Table 1. 


\begin{tabular}{|l|l|l|c|c|c|c|c|}
\hline No. & Site & Peptide sequence & $\mathbf{m} / \mathbf{z}$ & $\mathbf{z}$ & $\mathbf{p p m}$ & Score & Expect val. \\
\hline $1 \mathrm{a}^{*}$ & 17 & K(GlyGly)LPVEGSEEDK & 448.8929 & 3 & -0.003 & 43.0 & $8.9 \mathrm{e}-9$ \\
\hline $1 \mathrm{~b}^{*}$ & 17 & K(LeuArgGlyGly)LPVEGSEEDK & 538.6210 & 3 & -0.54 & 29.4 & $3.3 \mathrm{e}-6$ \\
\hline 2 & 37 & ASK(GlyGly)GGLIYGNYLHLEK & 626.3360 & 3 & 1.00 & 46.0 & $1.4 \mathrm{e}-5$ \\
\hline 3 & 62 & VLNAQELQSETK(GlyGly)GNK & 886.9619 & 2 & 1.60 & 52.5 & $2.2 \mathrm{e}-6$ \\
\hline $4^{*}$ & 110 & NMLK(GlyGly)VVSR & 530.8001 & 2 & -0.24 & 38.0 & $3.2 \mathrm{e}-3$ \\
\hline 5 & 164 & LLENK(GlyGly)IGVLQNMR & 547.9748 & 3 & -0.39 & 40.4 & $1.9 \mathrm{e}-5$ \\
\hline $6^{*}$ & 185 & DNFK(GlyGly)GEENELLLK & 831.9213 & 2 & 1.20 & 40.5 & $2.9 \mathrm{e}-5$ \\
\hline $7 \mathrm{a}^{*}$ & 194 & GEENELLLK(GlyGly)SEQEK & 880.4403 & 2 & 1.40 & 45.7 & $5.5 \mathrm{e}-5$ \\
\hline $7 \mathrm{~b}^{*}$ & 194 & DNFKGEENELLK(GlyGly)SEQEK & 755.3731 & 3 & 0.29 & 47.0 & $2.6 \mathrm{e}-4$ \\
\hline 8 & 228 & LEK(GlyGly)NITR & 494.2831 & 2 & 0.76 & 31.5 & $5.1 \mathrm{e}-4$ \\
\hline $9 \mathrm{a}$ & 244 & IQAK(GlyGly)EESEEKEEQVAEFQK & 798.3884 & 3 & 1.70 & 48.9 & $1.1 \mathrm{e}-7$ \\
\hline $9 \mathrm{~b}$ & 244 & IQAK(GlyGly)EESEEK & 652.8205 & 2 & 0.67 & 31.1 & $3.2 \mathrm{e}-3$ \\
\hline 10 & 250 & EESEEK(GlyGly)EEQVAEFQK & 651.6294 & 3 & 0.86 & 33.0 & $1.0 \mathrm{e}-4$ \\
\hline $11 \mathrm{a}^{*}$ & 259 & EESEEKEEQVAEFQK(GlyGly)QK & 737.0137 & 3 & 0.47 & 32.6 & $1.6 \mathrm{e}-4$ \\
\hline $11 \mathrm{~b}^{*}$ & 259 & EEQVAEFQK(GlyGly)QK & 739.3688 & 2 & 1.50 & 31.0 & $5.9 \mathrm{e}-4$ \\
\hline $12 \mathrm{a}$ & 271 & EVLLSLFDEK(GlyGly)R & 731.8986 & 2 & -0.06 & 37.8 & $8.1 \mathrm{e}-4$ \\
\hline $12 \mathrm{~b}$ & 271 & EVLLSLFDEK(LeuArgGlyGly)R & 577.9969 & 3 & 0.55 & 39.5 & $7.8 \mathrm{e}-8$ \\
\hline 13 & 279 & RHEHLLSK(GlyGly)GER & 492.5993 & 3 & 0.52 & 25.6 & $2.7 \mathrm{e}-3$ \\
\hline $14 \mathrm{a}$ & 339 & M(Oxidation)LGSK(GlyGly)AGTGGSSGYHYLR & 657.9835 & 3 & 0.92 & 57.1 & $2.5 \mathrm{e}-8$ \\
\hline $14 \mathrm{~b}$ & 339 & MLGSK(GlyGly)AGTGGSSGYHYLR & 978.4751 & 2 & 1.90 & 46.6 & $1.9 \mathrm{e}-8$ \\
\hline 15 & 380 & HWIPK(GlyGly)MNPTIHK & 539.2888 & 3 & -0.45 & 32.2 & $3.5 \mathrm{e}-4$ \\
\hline
\end{tabular}

Table 2. List of hTDO sites ubiquitinated by two E2/E3 systems, Ubc7p/gp78 and UbcH5a/CHIP\#. ${ }^{*}$ ewly identified in this work. \#These two E2-E3 systems were found to be major catalysts in in vitro reconstituted hTDO ubiquitination reactions.

The structure of hTDO was determined by the molecular replacement method, as implemented in the program MOLREP ${ }^{46}$, using the structure of XcTDO tetramer (PDB entry 2NW8) as the search model ${ }^{25}$. The atomic models were built with the program XtalView ${ }^{47}$, and the structure refinement was carried out with $\mathrm{CNS}^{48}$ and PHENIX $^{49}$. The refinement statistics are summarized in Table 1. Coordinates and structure factors have been deposited in the Protein Data Bank with accession codes 5 TI9 and 5TIA.

Mutagenesis. The mutants were made with the QuikChange kit (Stratagene) and verified by sequencing. The mutant proteins were expressed and purified following the same protocol as that for the wild-type protein.

Stopped-flow measurements. The experiments were performed by either mixing deoxy ferrous enzymes (final concentration $\sim 0.5$ or $3 \mu \mathrm{M}$ ) with air-saturated buffer or mixing ferric enzymes with buffer containing different concentrations of L-Trp in a $\pi^{\star} 180$ system from applied Photophysics Ltd (Leatherhead, Surrey, UK).

The deoxy ferrous enzyme was prepared by stoichiometrically titrating $\mathrm{N}_{2}$-purged ferric enzyme with dithionite. All the solutions were prepared in $\mathrm{pH} 8$ Tris buffer $(50 \mathrm{mM})$, containing $150 \mathrm{mM} \mathrm{NaCl}$ and a desired amount of Trp. The temperature was controlled at $25^{\circ} \mathrm{C}$ by a circulating water bath (Neslab RTE-9DD). For steady-state activity measurements, the formation of the product, NFK, was monitored at $321 \mathrm{~nm}\left(\varepsilon=3750 \mathrm{M}^{-1} \mathrm{~cm}^{-1}\right)$ as a function of time. The initial linear velocity of the reaction was plotted as a function of substrate concentration and fitted with the Michaelis-Menten equation using Origin 6.1 software (Microcal Software, Inc., MA, USA).

Isothermal titration calorimetry (ITC) measurements. The Trp affinities were measured in a Microcal VP-ITC (Northhampton, MA) in an anaerobic glove box. The ITC was calibrated using the built-in electrical calibration check. The ferric WT hTDO solution $(0.2 \mathrm{mM})$ in the reaction cell and the Trp solution $(3.5 \mathrm{mM})$ in the syringe were prepared in the same buffer in $\mathrm{ddH} 2 \mathrm{O}(\mathrm{pH} 8100 \mathrm{mM}$ Tris containing $150 \mathrm{mM} \mathrm{NaCl})$. All solutions were pre-purged with nitrogen gas, to avoid turnover. Following thermal equilibrium at $20^{\circ} \mathrm{C}$, an initial $600 \mathrm{~s}$ delay and a single $2.0 \mu \mathrm{l}$ titrant injection, 10 serial injections of $4 \mu \mathrm{l}$, followed by 40 serial injection of $6 \mu \mathrm{l}$ Trp solution, were done at an interval of $300 \mathrm{~s}$ into the stirred sample cell $(1.4 \mathrm{~mL})$ containing the ferric hTDO complex at a stirring rate of $155 \mathrm{rpm}$. A reference power of $15 \mu \mathrm{cal} / \mathrm{s}$ was used. The heat associated with each titration peak was integrated and plotted against the respective molar ratio of Trp/hTDO. Similar procedure was used for the measurement of the W208V/R211L mutant $(0.1 \mathrm{mM})$, except that following thermal equilibrium at $20^{\circ} \mathrm{C}$, an initial $600 \mathrm{~s}$ delay and a single $2.0 \mu \mathrm{l}$ titrant injection, 47 serial injections of $6 \mu \mathrm{l}$ Trp solution were done at an interval of $450 \mathrm{~s}$ into the stirred sample cell. Data were analyzed using nonlinear least-squares curve fitting in Origin7.0 (OriginLab Corp., Northampton, MA) using the standard one-binding site model.

${ }^{35}$ S-L-Met/Cys-pulse-chase analyses. HepG2 cells were transfected with the pcDNA6-hTDO-(His) ${ }_{6}$ or pcDNA6-EWR-(His) ${ }_{6}$ vector and grown to confluency in DMEM in 6-well-plates for $48 \mathrm{~h}$. The medium was then removed and replaced with methionine/cysteine-free DMEM containing $2.5 \mathrm{mM} \alpha$-methyltryptophan $(\alpha$ MTrp) for $1 \mathrm{~h}$. Subsequently, each well was pulsed with $75 \mu \mathrm{Ci}$ of ${ }^{35} \mathrm{~S}-\mathrm{L}$-Met/Cys for $1 \mathrm{~h}$. Each ${ }^{35} \mathrm{~S}$-labeled culture was then washed twice with ice-cold PBS containing $0.2 \mathrm{mM}$ methionine and $1.4 \mathrm{mM}$ cysteine, followed by 
DMEM containing $2.5 \mathrm{mM} \alpha \mathrm{MTrp}, 5 \mathrm{mM}$ cold methionine and cysteine, and further incubated for $0,30,60$ and $90 \mathrm{~min}$ at $37^{\circ} \mathrm{C}$. The cells harvested at each time point were lysed in Cell-Signaling Lysis buffer, containing general protease and phosphatase inhibitors, and $\mathrm{N}$-ethylmaleimide $(10 \mathrm{mM})$ to inhibit deubiquitinases. The lysates were centrifuged at $10,000 \mathrm{~g}$ at $4^{\circ} \mathrm{C}$ for $10 \mathrm{~min}$ to remove insoluble cell debris. The protein concentration was determined by the bicinchoninic assay (BCA). Lysate protein $(200 \mu \mathrm{g})$ was then diluted $(1: 4, \mathrm{v}: \mathrm{v})$ in Dynabead pull-down buffer and mixed with Dynabeads $(50 \mu \mathrm{l})$. The mixture was incubated at $4{ }^{\circ} \mathrm{C}$ with rotation overnight. The Dynabeads-His ${ }_{6}$-tagged hTDO protein complexes were then collected using a magnetic stand and washed five times with Dynabead-washing buffer. The $\mathrm{His}_{6}$-tagged hTDO proteins were eluted by heating the complexes for $5 \mathrm{~min}$ in an SDS-PAGE sample-loading buffer $(40 \mu \mathrm{l}, 62.5 \mathrm{mM} \mathrm{pH} 6.8$ Tris buffer containing $25 \%$ (v/v) glycerol, $10 \%(\mathrm{w} / \mathrm{v})$ SDS, $5 \%(\mathrm{v} / \mathrm{v}) \beta$-mercaptoethanol and $0.01 \%(\mathrm{w} / \mathrm{v})$ bromophenol blue). The radioactivity of a $10 \mu$ 1 aliquot was monitored in $4 \mathrm{ml}$ of Ecolume using a Beckman LS3801 liquid scintillation counter. The radioactivity of the eluates was used for the pulse-chase ${ }^{35} \mathrm{~S}$-TDO-degradation analysis. Another $30 \mu \mathrm{l}$-aliquot of the eluate (containing parent TDO protein and its ubiquitinated species) was subjected to SDS-PAGE (4-15\%). The gels were dried and subjected to fluorography with Typhoon scanning.

In vitro hTDO ubiquitination by 3 different E2/E3 Ub-ligase systems. Purified recombinant hTDO $(400 \mathrm{pmol})$ was incubated in each of the three functionally reconstituted E2/E3 systems in a final volume of $50 \mu \mathrm{l}$, and the reactions initiated with an ATP-regenerating system as described previously $y^{43,44,50}$. The reaction mixtures were incubated at $30^{\circ} \mathrm{C}$ for $60 \mathrm{~min}$. Aliquots $(30 \mu \mathrm{l})$ of each reaction mixture were then subjected to SDS-PAGE and Western immunoblotting analyses with subsequent 5-min exposure to electrochemiluminescent (ECL) substrate for pico-detection, as described ${ }^{44,50}$. The film was developed and visualized using a Typhoon scanner in the chemiluminescence mode.

Identification of hTDO K-ubiquitination sites. Ubiquitinated hTDO proteins generated as described above were combined and precipitated with 2 -volumes of ice-cold acetone at $-20^{\circ} \mathrm{C}$, overnight. The pellets were redissolved in $50 \mathrm{mM}$ ammonium bicarbonate $(\mathrm{ABC})$ solution containing $8 \mathrm{M}$ urea and reduced with tris (2-carboxyethyl) phosphine (TCEP, $10 \mathrm{mM})$ and then alkylated with chloroacetamide $(20 \mathrm{mM})$ at room temperature in the dark and diluted with the same $\mathrm{ABC}$ solution to a final concentration of $<2 \mathrm{M}$ urea followed by a combined lysyl endoprotease $\mathrm{C}$ (Lys-C)/trypsin digestion with a 1:25 enzyme:protein mass ratio, at $37^{\circ} \mathrm{C}$ for 16-18 $\mathrm{h}$. The digested peptides were desalted and extracted with a Sep-Pak C18 classic cartridge (Waters Inc.) and subjected to anti-KGG antibody (PTMScan Ubiquitin Remnant Motif kit, Cell Signaling) pull-down to enrich the ubiquitinated peptides before LC-MS/MS analyses. Ubiquitinated samples were analyzed on an LTQ-Orbitrap Velos mass spectrometer (Thermo Scientific) equipped with a nanoAcquity UPLC system (Waters). Peptides were resuspended in $0.1 \%$ formic acid and separated in an Easy-Spray column (Thermo, PepMap, C18, $3 \mu \mathrm{m}, 100 \AA$, $75 \mu \mathrm{m} \times 15 \mathrm{~cm})$ using a chromatographic system with a linear gradient from $2 \%$ solvent $\mathrm{A}(0.1 \%$ formic acid in water) to $35 \%$ solvent B ( $0.1 \%$ formic acid in acetonitrile $)$ at $300 \mathrm{~nL} / \mathrm{min}$ over $35 \mathrm{~min}$. MS precursor spectra were measured in the Orbitrap from $300-2000 \mathrm{~m} / z$ at 30,000 resolving power, selected and dissociated by higher energy collisional dissociation (HCD) for MS/MS analyses as described ${ }^{44,50}$. The MS/MS data were searched against the SwissProt database using the in-house Protein Prospector search engine (UCSF), with a concatenated database consisting of normal and randomized decoy databases. In addition to common modifications such as methionine oxidation and protein N-terminal acetylation, GlyGly (Uncleaved K), LeuArgGlyGly (Uncleaved K) modifications were considered for ubiquitination in the database search. False discovery rate (FDR) for ubiquitination was estimated to be $1 \%$ corresponding to the maximum expectation values of 0.01 . Peptides with expectation values of 0.01 or less were accepted.

\section{References}

1. Hayaishi, O. My life with tryptophan - never a dull moment. Prot. Sci. 2, 472-475 (1993).

2. Sono, M., Roach, M. P., Coulter, E. D. \& Dawson, J. H. Heme-containing oxygenases. Chem. Rev. 96, 2841-2887 (1996).

3. Takikawa, O. Biochemical and medical aspects of the indoleamine 2,3-dioxygenase-initiated L-tryptophan metabolism. Biochem. Biophys. Res. Commun. 338, 12-19 (2005).

4. Batabyal, D. \& Yeh, S.-R. Human tryptophan dioxygenase: a comparison to indoleamine 2,3-dioxygenase. J. Amer. Chem. Soc. 129, 15690-15701 (2007).

5. Khan, J. A., Forouhar, F., Tao, X. \& Tong, L. Nicotinamide adenine dinucleotide metabolism as attractive target for drug discovery. Expert Opin. Ther. Targets 11 (2007).

6. Efimov, I. et al. Structure and reaction mechanism in the heme dioxygenases. Biochem. 50, 2717-2724 (2011).

7. Poulos, T. L. Heme enzyme structure and function. Chem. Rev. 114, 3919-3962 (2014).

8. Searles, L. L. \& Voelker, R. A. Molecular characterization of the Drosophila vermillion locus and its suppressive alleles. Proc. Natl. Acad. Sci. USA 83, 404-408 (1986).

9. Kanai, M. et al. Tryptophan 2,3-dioxygenase is a key modulator of physiological neurogenesis and anxiety-related behavior in mice. Mol. Brain 2, 8 (2009).

10. Campesan, S. et al. The kynurenine pathway modulates neurodegeneration in a Drosophila model of Huntington's disease. Curr. Biol. 21, 961-966 (2011).

11. Godin-Ethier, J., Hanafi, L.-A., Piccirillo, C. A. \& Lapointe, R. Indoleamine 2,3-dioxygenase expression in human cancers: clinical and immunologic perspectives. Clin. Cancer Res. 17, 6985-6991 (2011).

12. von Bubnoff, D. \& Bieber, T. The indoleamine 2,3-dioxygenase (IDO) pathway controls allergy. Allergy 67, 718-725 (2012).

13. Munn, D. H. \& Mellor, A. L. Indoleamine 2,3 dioxygenase and metabolic control of immune responses. Trends Immunol. 34, 137-143 (2013)

14. Oxenkrug, G. Serotonin-kynurenine hypothesis of depression: historical overview and recent developments. Curr. Drug Targets 14, 514-521 (2013).

15. Vecsei, L., Szalardy, L., Fulop, F. \& Toldi, J. Kynurenines in the CNS: recent advances and new questions. Nat. Rev. Drug Discov. 12, 64-82 (2013) 
16. Ball, H. J., Jusof, F. F., Bakmiwewa, S. M., Hunt, N. H. \& Yuasa, H. J. Tryptophan-catabolizing enzymes - party of three. Front. Immunol. 5, 485 (2014).

17. Opitz, C. A. et al. An endogenous tumour-promoting ligand of the human aryl hydrocarbon receptor. Nature 478, 197-203 (2011).

18. Pilotte, L. et al. Reversal of tumoral immune resistance by inhibition of tryptophan 2,3-dioxygenase. Proc. Natl. Acad. Sci. USA 109, 2497-2502 (2012).

19. Predergast, G. C., Chang, M. Y., Mandik-Nayak, L., Metz, R. \& Muller, A. J. Indoleamine 2,3-dioxygenase as a modifier of pathogenic inflammation in cancer and other inflammation-associated diseases. Curr. Med. Chem. 18, 2257-2262 (2011).

20. Adams, S. et al. The kynurenine pathway in brain tumor pathogenesis. Cancer Res. 72, 5649-5657 (2012).

21. Austin, C. J. \& Rendina, L. M. Targeting key dioxygenases in tryptophan-kynurenine metabolism for immunomodulation and cancer chemotherapy. Drug Discov. Today 20, 609-617 (2015).

22. Platten, M., von Knebel Doeberitz, N., Oezen, I., Wick, W. \& Ochs, K. Cancer immunotherapy by targeting IDO1/TDO and their downstream effectors. Front. Immunol. 5, 673 (2015).

23. Dounay, A. B., Tuttle, J. B. \& Verhoest, P. R. Challenges and opportunities in the discovery of new therapeutics targeting the kynurenine pathway. J. Med. Chem. 58, 8762-8782 (2015).

24. Moon, Y. W., Hajjar, J., Hwu, P. \& Naing, A. Targeting the indoleamine 2,3-dioxygenase pathway in cancer. J. Immunother. Cancer 3, $51(2015)$.

25. Forouhar, F. et al. Molecular insights into substrate recognition and catalysis by tryptophan 2,3-dioxygenase. Proc. Natl. Acad. Sci. USA 104, 473-478 (2007).

26. Zhang, Y. et al. Crystal structure and mechanism of tryptophan 2,3-dioxygenase, a heme enzyme involved in tryptophan catabolism and in quinolinate biosynthesis. Biochem. 46, 145-155 (2007).

27. Huang, W., Gong, Z., Li, J. \& Ding, J. Crystal structure of Drosophila melanogaster tryptophan 2,3-dioxygenase reveals insights into substrate recognition and catalytic mechanism. J. Struct. Biol. (2013).

28. Meng, B. et al. Structural and functional analyses of human tryptophan 2,3-dioxygenase. Proteins 82, 3210-3216 (2014).

29. Sugimoto, H. et al. Crystal structure of human indoleamine 2,3-dioxygenase: Catalytic mechnaism of $\mathrm{O} 2$ incorporation by a hemecontaining dioxygenase. Proc. Natl. Acad. Sci. USA 103, 2611-2616 (2006).

30. Rovira, C. \& Parrinello, M. Harmonic and anharmonic dynamics of Fe-CO and Fe-O2 in heme models. Biophys. J. 78, 93-100 (2000).

31. Lewis-Ballester, A. et al. Evidence for a ferryl intermediate in a heme-based dioxygenase. Proc. Natl. Acad. Sci. USA 106, 17371-17376 (2009).

32. Chung, L. W., Li, X., Sugimoto, H., Shiro, Y. \& Morokuma, K. ONIOM study on a missing piece in our understanding of heme chemistry: bacterial tryptophan 2,3-dioxygenase with dual oxidants. J. Amer. Chem. Soc. 132, 11993-12005 (2010).

33. Capece, L., Lewis-Ballester, A., Yeh, S.-R., Estrin, D. A. \& Marti, M. A. Complete reaction mechanism of indoleamine 2,3-dioxygenase as revealed by QM/MM simulations. J. Phys. Chem. B 116, 1401-1413 (2012).

34. Davydov, R. M. et al. Probing the ternary complexes of indoleamine and tryptophan 2,3-dioxygenases by cryoreduction EPR and ENDOR spectroscopy. J. Amer. Chem. Soc. 132, 5494-5500 (2010).

35. Thackray, S. J. et al. Histidine 55 fo trytophan 2,3-dioxygenase is not an active site base but regulates catalysis by controlling substrate binding. Biochem. 47, 10677-10684 (2008).

36. Batabyal, D. \& Yeh, S.-R. Substrate-protein interaction in human tryptophan dioxygenase: the critical role of H76. J. Amer. Chem. Soc. 131, 3260-3270 (2009).

37. Gupta, R., Fu, R., Liu, A. \& Hendrich, M. P. EPR and Mossbauer spectroscopy show inequivalent hemes in tryptophan dioxygenase. J. Amer. Chem. Soc. 132, 1098-1109 (2010).

38. Basran, J. et al. The mechanism of formation of N-formylkynurenine by heme dioxygenases. J. Amer. Chem. Soc. 133, 16251-16257 (2011).

39. Makino, R. et al. Initial O2 insertion step of the tryptophan dioxygenase reaction proposed by a heme-modification study. Biochem. 54, 3604-3616 (2015).

40. Chung, L. W., Li, X., Sugimoto, H., Shiro, Y. \& Morokuma, K. Density functional theory study on a missing piece in understanding of heme chemistry: the reaction mechanism for indoleamine 2,3-dioxygenase and tryptophan 2,3-dioxygenase. J. Amer. Chem. Soc. 130, 12299-12309 (2008).

41. Schimke, R. T. \& Doyle, D. Control of enzyme levels in animal tissues. Ann. Rev. Biochem. 39, 929-976 (1970).

42. Wagner, S. A. et al. Proteomic analyses reveal divergent ubiquitylation site patterns in murine tissues. Mol. Cell. Proteomics 11, 1578-1585 (2012).

43. Pabarcus, M. et al. CYP3A4 ubiquitination by gp78 (the tumor autocrine motility factor receptor, AMFR) and CHIP E3 ligases. Arch. Biochem. Biophys. 483, 66-74 (2009).

44. Wang, Y. Q. et al. Human liver cytochrome P450 3A4 ubiquitination: molecular recognition by UBC7-gp78 autocrine motility factor receptor (AMFR) and UbcH5a-CHIP-Hsc70-Hsp40 E2-E3 ubiquitin ligase complexes. J. Biol. Chem. 290, 3308-3332 (2015).

45. Otwinowski, Z. \& Minor, W. Processing of X-ray diffraction data collected in oscillation mode. Method Enzymol. 276, 307-326 (1997).

46. Vagin, A. A. \& Teplyakov, A. An approach to multi-copy search in molecular replacement. Acta Cryst. D56, 1622-1624 (2000).

47. McRee, D. E. XtalView/Xfit-a versatile program for manipulating atomic coordinates and electron density. J. Struct. Biol. 125, 156-165 (1999).

48. Brunger, A. T. et al. Crystallography \& NMR System: A new software suite for macromolecular structure determination. Acta Cryst. D54, 905-921 (1998).

49. Adams, P. D. et al. PHENIX: building a new software for automated crystallographic structure determination. Acta Cryst. D58, 1948-1954 (2002).

50. Wang, Y. Q. et al. Ubiquitin-dependent proteasomal degradation (UPD) of human liver cytochrome P450 2E1: identification of sites targeted for phosphorylation and ubiquitination. J. Biol. Chem. 286, 9443-9456 (2011).

\section{Acknowledgements}

We thank Ron Breslow for helpful discussions; Randy Abramowitz and John Schwanof at the NSLS for setting up the X4A and X4C beamlines; Angela Lauricella and George DeTitta of Hauptman-Woodward Research Institute for crystallization screening; Drs. Shenheng Guan and Michael Trnka, UCSF, for LC-MS/MS analyses of hTDO ubiquitination. This research was supported by grants from the Protein Structure Initiative of the National Institutes of Health (P50 GM62413 and U54 GM074958) to LT, National Institutes of Health Grants GM086482 and GM115773 to SRY, GM44037 and DK26506 to MAC, and NIH NIGMS 8P41GM103481 support of the UCSF Bio-Organic Biomedical Mass Spectrometry Resource at UCSF (A. L. Burlingame, Director). 


\section{Author Contributions}

S.-R.Y. conceived microscopic spectroscopy-guided crystallography studies. D.B., A.L.-B. and S.K. expressed and purified recombinant hTDO. M.H., S.L. and F.F. created a reproducible crystallization protocol for ferric and ferrous hTDO complexes, which was used by A.L.-B. to produce crystals of deoxy ferrous hTDO. J.S. performed $\mathrm{X}$-ray diffraction screening, data collection and processing. F.F. determined and refined the structures. A.L.-B. carried out biochemical and biophysical studies in free solution. S.-R.Y., A.L.-B. and S.K. carried out microscopic spectroscopy studies. S.-R.Y. and A.L.-B. conceived Y175 and NFK-release studies. S.-R.Y., M.A.C. and B.Y.C. conceived exo site and UPD studies. S.M.K. and Y.Q.W. carried out cellular and in vitro UPD studies, respectively. S.K. prepared plasmids and protein samples for UPD studies. B.Y.C. contributed initial construction of plasmids for UPD studies. L.T. and F.F. analyzed the diffraction data. S.-R.Y. and A.L.-B. analyzed the biochemical and biophysical data. M.A.C., S.-M.K. and Y.Q.W. analyzed the UPD-related data. L.T., S.-R.Y., M.A.C., A.L.-B., F.F., S.-M.K. and Y.Q.W. contributed to writing the manuscript, and all authors commented on the manuscript.

\section{Additional Information \\ Supplementary information accompanies this paper at http://www.nature.com/srep}

Competing financial interests: The authors declare no competing financial interests.

How to cite this article: Lewis-Ballester, A. et al. Molecular basis for catalysis and substrate-mediated cellular stabilization of human tryptophan 2,3-dioxygenase. Sci. Rep. 6, 35169; doi: 10.1038/srep35169 (2016).

(c) (i) This work is licensed under a Creative Commons Attribution 4.0 International License. The images or other third party material in this article are included in the article's Creative Commons license, unless indicated otherwise in the credit line; if the material is not included under the Creative Commons license, users will need to obtain permission from the license holder to reproduce the material. To view a copy of this license, visit http://creativecommons.org/licenses/by/4.0/

(C) The Author(s) 2016 